\title{
Physiological Changes and Shelf-Life Extension of Papaya (Carica papaya L.) cv. Red Lady as Influenced by Pre-Harvest Spray of Plant Elicitors
}

\author{
Sreedhar Devarakonda ${ }^{1 *}$, C. Madhumathi ${ }^{1}$, V. Umamahesh ${ }^{2}$, L. Mukunda Lakshmi ${ }^{3}$, \\ M. Lakshmi Narayana Reddy ${ }^{4}$, V. Vijaya Bhaskar ${ }^{5}$ and T. Rajasekharam ${ }^{3}$
}

\author{
${ }^{1}$ Horticultural Research Station, Dr YSRHU, Anantharajupeta, Andhra Pradesh, India \\ ${ }^{2}$ Department of Plant Physiology, College of Agriculture, \\ ANGRAU, Tirupati, Andhra Pradesh, India \\ ${ }^{3}$ Citrus Research Station, Dr YSRHU, Tirupathi, Andhra Pradesh, India \\ ${ }^{4} \mathrm{Dr}$ YSRHU, VR Gudem, Andhra Pradesh, India \\ ${ }^{5}$ Department of Floriculture and Landscaping, College of Horticulture, \\ Dr YSRHU, Anantharajupeta, Andhra Pradesh, India
}

\section{*Corresponding author}

\section{A B S T R A C T}

\begin{tabular}{|l|}
\hline K e y w o r d s \\
Papaya, Salicylic \\
acid, Jasmonic \\
Acid, Physical \\
Characters \\
\hline Article Info \\
\hline $\begin{array}{l}\text { Accepted: } \\
18 \text { April } 2020 \\
\text { Available Online: } \\
\text { 10 May } 2020\end{array}$ \\
\hline
\end{tabular}

To study the effect of pre-harvest spray of plant elicitors at flowering on fruit characters and postharvest behaviour of papaya, was planned at Horticultural Research station, Anantharajupeta, Railway Kodur, Andhra Pradesh during 2015-16 and 2016-17 with the application of salicylic acid (50 ppm, $100 \mathrm{ppm}, 150 \mathrm{ppm})$, jasmonic acid $(50 \mu \mathrm{M}, 100 \mu \mathrm{M}, 150 \mu \mathrm{M})$ once at 50 Days After Anthesis and twice at 50 Days After Anthesis and 75 Days After Anthesis and control (no spray). The data revealed that $\mathrm{T}_{9}$ (SA @ $150 \mathrm{ppm}$ at 50 and $75 \mathrm{DAA}$ ) recorded highest fruit weight (1.03 $\mathrm{kg}$ ), fruit length $(17.89 \mathrm{~cm})$, fruit girth $(41.34 \mathrm{~cm})$, fruit volume $(1.38$ liters $)$, cavity length $(12.64$ $\mathrm{cm})$, cavity width $(7.37 \mathrm{~cm})$, weight of the pulp $(0.87 \mathrm{~kg})$ and flesh thickness $(2.95 \mathrm{~cm}) . \mathrm{T}_{8}$ (SA @ $100 \mathrm{ppm}$ at 50 and $75 \mathrm{DAA}$ ) and $\mathrm{T}_{7}$ (SA @ $50 \mathrm{ppm}$ at 50 and $75 \mathrm{DAA}$ ) were observed to be on par with $\mathrm{T}_{9}$ in majority of the cases with respect to these fruit physical characters. The corresponding lowest values in this regard were recorded with $\mathrm{T}_{13}$ (control). The positive influence of $\mathrm{T}_{9}$ and $\mathrm{T}_{8}$ was also observed on increased shelf life of papaya fruits (10.5 and 10.17 days respectively). However, the fruits obtained from untreated plants $\left(\mathrm{T}_{13}\right)$ recorded minimum shelf life (6.17 days). The quality attribute such as T.S.S. showed an increasing trend up to 9 days after storage and decreased further.

\section{Introduction}

Papaya (Carica papaya L.) belongs to the genus Carica, of the family Caricaceae. Among the 48 species of this family, it is the most cultivated species and commonly called as papaw or paw paw (Australia), mamao (Brazil) and tree melon (China). It is cultivated in the world in an area of 0.44 million ha with production of 12.67 million MT. In India it is grown in an area of $1,36,100$ ha and annual production of 6.10 
million MT with a productivity of $44.9 \mathrm{t} \mathrm{ha}^{-1}$. Papaya in India is the highest producer occupying 44.4 per cent of world's papaya production. We are exporting of about 12,773 MT of papaya to different countries specially United Arab Emirates, Saudi Arabia, Netherland, Bahrain, Qutar, Omen, Nepal, US and Germany (Indiastat, 2016-17). So, there is need of extending the shelf life period of papaya as it is transported to very distant markets. Jasmonic acid (JA) and Salicylic acid (SA) are two endogenous signalling molecules used in regulation of plant resistance to pathogens and herbivores (Farousk and Osman, 2011). Further, it was reported that SA and JA plays an important role in maintaining the shelf life and improving post-harvest quality of the fruit. Hence, SA and JA were used as preharvest spray to extend the shelflife of papaya. The pattern of fruit development in papaya follows a double sigmoid type of growth curve with definite lag phase. Physiologically papaya is a climacteric fruit with typical respiratory peak and ethylene production patterns during ripening. Among several postharvest quality problems in papaya, pulp lumpiness (uneven ripening), decay and excessive pulp firmness at the edible stage are some crucial parameters to be taken care of. It is also true that the extremely delicate nature of papaya fruits causes more weight loss and heavy spoilage during transport before it reaches to the consumer.

Salicylic acid is reported to be directly toxic to fungi as it significantly inhibits fungal growth and spore germination of the pathogen in vitro. It can delay ripening of fruits, probably through inhibition of ethylene biosynthesis or action.

Besides methyl jasmonate also extends shelf life and reduce microbial contamination of fresh-cut celery and peppers (Buta and Moline, 1998).

\section{Materials and Methods}

The experiment was carried out at Horticultural Research Station, Anantharajupeta, Kadapa district, Andhra Pradesh during the period from November, 2015 to October, 2016 and November, 2016 to October, 2017. The experiment was laid out in a randomized block design with thirteen treatments and three replications. The treatments tested were application of salicylic acid@ $@ 50$ ppm $\left(\mathrm{T}_{1}\right), 100$ ppm $\left(\mathrm{T}_{2}\right), 150$ ppm $\left(\mathrm{T}_{3}\right)$ at 50DAA, jasmonic acid @ $50 \mu \mathrm{M}\left(\mathrm{T}_{4}\right)$, $100 \mu \mathrm{M}\left(\mathrm{T}_{5}\right), 150 \mu \mathrm{M}\left(\mathrm{T}_{6}\right)$ at 50DAA, salicylic acid @ 50 ppm $\left(\mathrm{T}_{7}\right), 100$ ppm $\left(\mathrm{T}_{8}\right)$, $150 \mathrm{ppm}\left(\mathrm{T}_{9}\right)$ at 50 DAA and 75 DAA, jasmonic acid @ $50 \mu \mathrm{M}\left(\mathrm{T}_{10}\right), 100 \mu \mathrm{M}\left(\mathrm{T}_{11}\right)$, $150 \mu \mathrm{M}\left(\mathrm{T}_{12}\right)$ at $50 \mathrm{DAA}$ and 75 DAA and control $\left(\mathrm{T}_{13}\right)$.

Fruits were harvested from tagged treatmental plants which were sprayed with different concentration of salicylic acid and jasmonic acid at 50 and 75 DAA. This experiment was carried out with full mature green papaya fruits collected from each treatment separately. The fruits were separated into healthy marketable fruits, disease fruits and unmarketable fruits. Experimental material was selected randomly from the lot of healthy marketable fruits. But, Fruit characters studied by taking averages of the randomly selected plants. Fruits were harvested at colour break stage when green colour changed to light green with slight yellowish tinge at blossom end. Observations on the physico-chemical characteristics were recorded for every 3 days after harvesting of fruits. These fruits were kept for storage at ambient temperatures.

\section{Results and Discussion}

The fruit characters such as Fruit weight $(\mathrm{kg})$, Fruit length $(\mathrm{cm})$, Fruit girth $(\mathrm{cm})$ and Fruit volume (Litre) were presented in Table 1. 


\section{Fruit weight (kg)}

In pooled mean data the maximum fruit weight $(1.03 \mathrm{~kg})$ was noticed in $\mathrm{T}_{9}$ (SA @ $150 \mathrm{ppm}$ at 50 and 75 DAA) and it was on a par with $\mathrm{T}_{8}(1.00 \mathrm{~kg})$.

\section{Fruit length (cm)}

During second year and pooled mean data, the maximum fruit length was observed in $\mathrm{T}_{9}$ (SA @ $150 \mathrm{ppm}$ at 50 and 75 DAA) (17.67 and $17.89 \mathrm{~cm}$ respectively), which was statistically on a par with $\mathrm{T}_{8}(17.33$ and 17.60 $\mathrm{cm}$ respectively).

\section{Fruit girth (cm)}

Highest fruit girth $(41.34 \mathrm{~cm})$ was recorded in $\mathrm{T}_{8}$ (SA @ $100 \mathrm{ppm}$ at 50 and $75 \mathrm{DAA}$ ) than all other treatments except $\mathrm{T}_{9}(41.23 \mathrm{~cm}), \mathrm{T}_{7}$ $(39.48 \mathrm{~cm})$ and $\mathrm{T}_{10}(39.17 \mathrm{~cm})$ which were at par with $\mathrm{T}_{8}$ in pooled data.

\section{Fruit volume (L)}

The data from both the years and its pooled mean revealed that, application of $\mathrm{T}_{9}$ (SA @ $150 \mathrm{ppm}$ at 50 and 75 DAA) recorded maximum fruit volume $(1.45,1.31$ and 1.38 litre respectively), which was comparable with that of $\mathrm{T}_{8}(1.31,1.26$ and 1.28 litre respectively) and $\mathrm{T}_{7}$ (1.34, 1.22 and 1.28 litre respectively).

\section{Cavity length $(\mathrm{cm})$}

Highest cavity length $(12.64 \mathrm{~cm})$ in $\mathrm{T}_{9}$ (SA @ $150 \mathrm{ppm}$ at 50 and 75 DAA) which was at par with $\mathrm{T}_{8}(12.43 \mathrm{~cm})$ was observed in pooled data (Table 2).

\section{Cavity width (cm)}

Regarding pooled data, highest cavity width was observed in $\mathrm{T}_{8}$ (SA @ 100 ppm at 50 and
75 DAA) $(7.37 \mathrm{~cm})$ and it was on par with $\mathrm{T}_{9}$ $(7.31 \mathrm{~cm}), \mathrm{T}_{7}(7.17 \mathrm{~cm})$ and $\mathrm{T}_{10}(7.14 \mathrm{~cm})$ (Table 2).

\section{Cavity index (\%)}

In pooled mean data, pre harvest spray of salicylic acid@150 ppm at 50 and 75 DAA) $\left(\mathrm{T}_{9}\right), \mathrm{T}_{8}$ and $\mathrm{T}_{7}$ recorded minimum percentage of cavity index (19.11, 19.30 and $19.61 \%$ respectively) in papaya fruit and found at a par (Table 2).

\section{Weight of pulp (kg)}

Maximum pulp weight $(0.87 \mathrm{~kg})$ was observed in $\mathrm{T}_{9}$ (SA @ 150 ppm at 50 and 75 DAA), and it was statistically at a par with $\mathrm{T}_{8}$ $(0.85 \mathrm{~kg})$ and $\mathrm{T}_{7}(0.84 \mathrm{~kg})$ in pooled data (Table 3).

\section{Flesh thickness (cm) (pulp thickness)}

In second year of study, $\mathrm{T}_{9}(2.76 \mathrm{~cm}), \mathrm{T}_{8}$ $(2.67 \mathrm{~cm})$ and $\mathrm{T}_{7}(2.64 \mathrm{~cm})$ recorded significantly maximum pulp thickness and all are comparable with each other. Moreover, in pooled mean also almost the same trend was observed (Table 3 ).

In the present study foliar application of salicylic acid at increased concentrations at 50 and 75 DAA showed a positive effect in increasing fruit weight, fruit length, fruit volume, fruit girth, cavity length, cavity width and reduction in cavity index.

The increased fruit weight in present study by application of salicylic acid might be due to Salicylic acid enhances the biosynthesis of natural hormones, carbohydrates and stimulates cell division, enhances tolerance of plants to all stresses namely diseases, salt and water stresses and also protects plant cells from oxidation by production of free radicals (Raskin, 1992). 
Table.1 Effect of pre-harvest spray of salicylic acid and Jasmonic acid on fruit characters in papaya

\begin{tabular}{|c|c|c|c|c|c|c|c|c|c|c|c|c|}
\hline \multirow[t]{3}{*}{ Treatments } & \multicolumn{12}{|c|}{ Fruit characters } \\
\hline & \multicolumn{3}{|c|}{ Fruit weight (kg) } & \multicolumn{3}{|c|}{ Fruit length $(\mathrm{cm})$} & \multicolumn{3}{|c|}{ Fruit girth (cm) } & \multicolumn{3}{|c|}{ Fruit volume (Litre) } \\
\hline & $\begin{array}{c}\text { I year } \\
2015-16\end{array}$ & $\begin{array}{c}\text { II year } \\
\text { 2016-17 }\end{array}$ & $\begin{array}{c}\text { Pooled } \\
\text { data }\end{array}$ & $\begin{array}{c}\text { I year } \\
2015-16\end{array}$ & $\begin{array}{l}\text { II year } \\
\text { 2016-17 }\end{array}$ & $\begin{array}{c}\text { Pooled } \\
\text { data }\end{array}$ & $\begin{array}{l}\text { I year } \\
2015-16\end{array}$ & $\begin{array}{l}\text { II year } \\
2016-17\end{array}$ & $\begin{array}{c}\text { Pooled } \\
\text { data }\end{array}$ & $\begin{array}{c}\text { I year } \\
2015-16\end{array}$ & $\begin{array}{l}\text { II year } \\
2016-17\end{array}$ & $\begin{array}{c}\text { Pooled } \\
\text { data }\end{array}$ \\
\hline $\begin{array}{l}T_{1} \text { : Salicylic acid @ } \\
50 \text { ppm at } 50 \text { DAA }\end{array}$ & 0.83 & 0.87 & 0.85 & 16.33 & 16.73 & 16.53 & 36.51 & 37.48 & 36.99 & 1.10 & 1.13 & 1.12 \\
\hline $\begin{array}{l}T_{2} \text { : Salicylic acid @ } \\
100 \text { ppm at } 50 \text { DAA }\end{array}$ & 0.84 & 0.89 & 0.86 & 17.48 & 16.63 & 17.06 & 36.85 & 37.71 & 37.28 & 1.13 & 1.14 & 1.13 \\
\hline $\begin{array}{l}T_{3} \text { : Salicylic acid @ } \\
150 \text { ppm at } 50 \text { DAA }\end{array}$ & 0.97 & 0.91 & 0.94 & 17.57 & 16.90 & 17.23 & 39.74 & 38.09 & 38.91 & 1.28 & 1.19 & 1.23 \\
\hline $\begin{array}{l}\mathrm{T}_{4}: \text { Jasmonic acid } \\
@ 50 \mu \mathrm{M} \text { at } 50 \text { DAA }\end{array}$ & 0.83 & 0.84 & 0.84 & 17.47 & 16.52 & 16.99 & 37.27 & 35.19 & 36.23 & 1.08 & 1.15 & 1.12 \\
\hline $\begin{array}{l}T_{5}: \text { Jasmonic acid } \\
@ 100 \mu \mathrm{M} \text { at } 50 \mathrm{DAA}\end{array}$ & 0.90 & 0.86 & 0.88 & 16.80 & 16.81 & 16.81 & 37.76 & 37.53 & 37.65 & 1.18 & 1.13 & 1.15 \\
\hline $\begin{array}{l}\mathrm{T}_{6}: \text { Jasmonic acid } \\
@ 150 \mu \mathrm{M} \text { at } 50 \mathrm{DAA}\end{array}$ & 0.81 & 0.85 & 0.83 & 15.96 & 16.69 & 16.33 & 34.13 & 36.59 & 35.36 & 0.99 & 1.06 & 1.02 \\
\hline $\begin{array}{l}T_{7}: \text { Salicylic acid @ } \\
50 \text { ppm at } 50 \text { DAA } \\
\text { and } 75 \text { DAA }\end{array}$ & 0.99 & 0.96 & 0.97 & 17.65 & 17.19 & 17.42 & 39.36 & 39.61 & 39.48 & 1.34 & 1.22 & 1.28 \\
\hline $\begin{array}{l}T_{8}: \text { Salicylic acid @ } \\
100 \text { ppm at } 50 \text { DAA } \\
\text { and } 75 \text { DAA }\end{array}$ & 1.01 & 0.98 & 1.00 & 17.88 & 17.33 & 17.60 & 42.25 & 40.43 & 41.34 & 1.31 & 1.26 & 1.28 \\
\hline $\begin{array}{l}T_{9}: \text { Salicylic acid @ } \\
150 \text { ppm at } 50 \text { DAA } \\
\text { and } 75 \text { DAA }\end{array}$ & 1.05 & 1.01 & 1.03 & 18.10 & 17.67 & 17.89 & 41.35 & 41.11 & 41.23 & 1.45 & 1.31 & 1.38 \\
\hline $\begin{array}{l}\mathrm{T}_{10} \text { : Jasmonic acid } \\
\text { @ } 50 \mu \mathrm{M} \text { at } 50 \text { DAA } \\
\text { and } 75 \text { DAA }\end{array}$ & 0.91 & 0.90 & 0.90 & 17.64 & 16.94 & 17.29 & 39.23 & 39.12 & 39.17 & 1.20 & 1.15 & 1.17 \\
\hline $\begin{array}{l}T_{11}: \text { Jasmonic acid } \\
\text { @ } 100 \mu \mathrm{M} \text { at } 50 \text { DAA } \\
\text { and } 75 \text { DAA }\end{array}$ & 0.92 & 0.87 & 0.90 & 17.35 & 17.00 & 17.17 & 37.83 & 38.01 & 37.92 & 1.10 & 1.10 & 1.10 \\
\hline $\begin{array}{l}\mathrm{T}_{12}: \text { Jasmonic acid } \\
\text { @ } 150 \mu \mathrm{M} \text { at } 50 \text { DAA } \\
\text { and } 75 \mathrm{DAA}\end{array}$ & 0.89 & 0.88 & 0.88 & 16.62 & 16.85 & 16.74 & 38.72 & 37.56 & 38.14 & 1.15 & 1.13 & 1.14 \\
\hline $\mathbf{T}_{13}:$ Control & 0.78 & 0.81 & 0.80 & 15.78 & 16.11 & 15.95 & 33.96 & 34.59 & 34.27 & 0.87 & 0.97 & 0.92 \\
\hline S.Em. \pm & 0.02 & 0.02 & 0.01 & 0.16 & 0.16 & 0.11 & 1.37 & 1.07 & 0.92 & 0.08 & 0.04 & 0.04 \\
\hline C.D. at $5 \%$ & 0.06 & 0.05 & 0.03 & 0.48 & 0.47 & 0.31 & 4.01 & 3.12 & 2.68 & 0.22 & 0.12 & 0.12 \\
\hline
\end{tabular}

DAA: Days after anthesis 
Table.2 Effect of salicylic acid and jasmonic acid on cavity parameters of papaya

\begin{tabular}{|c|c|c|c|c|c|c|c|c|c|}
\hline \multirow[t]{2}{*}{ Treatments } & \multicolumn{3}{|c|}{ Cavity length $(\mathrm{cm})$} & \multicolumn{3}{|c|}{ Cavity width $(\mathrm{cm})$} & \multicolumn{3}{|c|}{ Cavity index (\%) } \\
\hline & $\begin{array}{l}\text { I year } \\
\text { 2015-16 }\end{array}$ & $\begin{array}{l}\text { II year } \\
\text { 2016-17 }\end{array}$ & $\begin{array}{c}\text { Pooled } \\
\text { data }\end{array}$ & $\begin{array}{c}\text { I year } \\
2015-16\end{array}$ & $\begin{array}{l}\text { II year } \\
2016-17\end{array}$ & $\begin{array}{c}\text { Pooled } \\
\text { data }\end{array}$ & $\begin{array}{l}\text { I year } \\
2015-16\end{array}$ & $\begin{array}{l}\text { II year } \\
\text { 2016-17 }\end{array}$ & $\begin{array}{c}\text { Pooled } \\
\text { data }\end{array}$ \\
\hline$T_{1}$ : Salicylic acid @ 50 ppm B.I. & 11.50 & 11.78 & 11.64 & 6.76 & 6.73 & 6.75 & 26.44 & 25.38 & 25.91 \\
\hline $\begin{array}{l}T_{2}: \text { Salicylic acid @ } 100 \text { ppm } \\
\text { B.I. }\end{array}$ & 11.90 & 11.89 & 11.90 & 6.19 & 6.65 & 6.42 & 25.71 & 24.88 & 25.30 \\
\hline $\begin{array}{l}T_{3}: \text { Salicylic acid @ } 150 \text { ppm } \\
\text { B.I. }\end{array}$ & 11.95 & 11.66 & 11.80 & 6.88 & 7.00 & 6.94 & 20.76 & 22.94 & 21.85 \\
\hline $\mathbf{T}_{4}$ : Jasmonic acid @ $50 \mu \mathrm{M}$ B.I. & 11.95 & 12.00 & 11.98 & 6.85 & 6.89 & 6.87 & 27.39 & 24.52 & 25.96 \\
\hline $\begin{array}{l}\mathrm{T}_{5}: \text { Jasmonic acid @ } 100 \mu \mathrm{M} \\
\text { B.I. }\end{array}$ & 11.73 & 12.09 & 11.91 & 6.27 & 6.26 & 6.27 & 23.01 & 24.37 & 23.69 \\
\hline $\begin{array}{l}\mathrm{T}_{6}: \text { Jasmonic acid @ } 150 \mu \mathrm{M} \\
\text { B.I. }\end{array}$ & 11.25 & 11.58 & 11.41 & 6.41 & 6.77 & 6.59 & 26.51 & 26.53 & 26.52 \\
\hline $\mathbf{T}_{7}$ : Salicylic acid @ 50 ppm A.I. & 11.90 & 11.92 & 11.91 & 7.43 & 6.90 & 7.17 & 18.83 & 20.39 & 19.61 \\
\hline $\begin{array}{l}\mathbf{T}_{8}: \text { Salicylic acid @ } 100 \text { ppm } \\
\text { A.I. }\end{array}$ & 12.65 & 12.21 & 12.43 & 7.52 & 7.22 & 7.37 & 19.23 & 19.37 & 19.30 \\
\hline $\begin{array}{l}T_{9}: \text { Salicylic acid @ } 150 \text { ppm } \\
\text { A.I. }\end{array}$ & 12.80 & 12.49 & 12.64 & 7.48 & 7.15 & 7.31 & 18.69 & 19.53 & 19.11 \\
\hline $\mathrm{T}_{10}:$ Jasmonic acid @ $50 \mu \mathrm{M}$ A.I. & 12.39 & 12.03 & 12.21 & 7.16 & 7.12 & 7.14 & 22.51 & 24.54 & 23.53 \\
\hline $\begin{array}{l}\mathrm{T}_{11}: \text { Jasmonic acid @ } 100 \mu \mathrm{M} \\
\text { A.I. }\end{array}$ & 11.96 & 11.97 & 11.97 & 6.54 & 6.39 & 6.47 & 25.95 & 26.49 & 26.22 \\
\hline $\begin{array}{l}T_{12}: \text { Jasmonic acid @ } 150 \mu M \\
\text { A.I. }\end{array}$ & 11.82 & 12.11 & 11.96 & 6.53 & 6.31 & 6.42 & 24.71 & 25.94 & 25.33 \\
\hline $\mathbf{T}_{13}:$ Control & 11.05 & 11.28 & 11.17 & 5.92 & 6.62 & 6.27 & 29.53 & 27.39 & 28.46 \\
\hline S.Em. \pm & 0.14 & 0.11 & 0.10 & 0.16 & 0.09 & 0.09 & 0.63 & 0.66 & 0.43 \\
\hline C.D. at $5 \%$ & 0.41 & 0.32 & 0.28 & 0.45 & 0.27 & 0.27 & 1.85 & 1.93 & 1.26 \\
\hline
\end{tabular}

B.I. :Before inoculation; A.I. : After inoculation; DAI : Days after inoculation 
Table.3 Effect of pre-harvest spray of salicylic acid and jasmonic acid on weight of pulp (kg) and flesh thickness (cm) in papaya

\begin{tabular}{|c|c|c|c|c|c|c|}
\hline \multirow[t]{2}{*}{ Treatments } & \multicolumn{3}{|c|}{ Weight of pulp (kg) } & \multicolumn{3}{|c|}{ Flesh thickness (cm) } \\
\hline & $\begin{array}{c}\text { I year } \\
2015-16\end{array}$ & $\begin{array}{c}\text { II year } \\
\text { 2016-17 }\end{array}$ & $\begin{array}{c}\text { Pooled } \\
\text { data }\end{array}$ & $\begin{array}{c}\text { I year } \\
2015-16\end{array}$ & $\begin{array}{c}\text { II year } \\
2016-17\end{array}$ & $\begin{array}{c}\text { Pooled } \\
\text { data }\end{array}$ \\
\hline $\mathbf{T}_{1}$ : Salicylic acid @ 50 ppm at 50 DAA & 0.72 & 0.76 & 0.74 & 2.10 & 2.26 & 2.18 \\
\hline$T_{2}:$ Salicylic acid @ 100 ppm at 50 DAA & 0.71 & 0.75 & 0.73 & 1.97 & 2.51 & 2.24 \\
\hline$T_{3}$ : Salicylic acid @ 150 ppm at 50 DAA & 0.82 & 0.76 & 0.79 & 2.28 & 2.56 & 2.42 \\
\hline $\mathrm{T}_{4}$ : Jasmonic acid @ $50 \mu \mathrm{M}$ at 50 DAA & 0.69 & 0.69 & 0.69 & 2.33 & 2.36 & 2.34 \\
\hline$T_{5}:$ Jasmonic acid @ $100 \mu \mathrm{M}$ at 50 DAA & 0.76 & 0.72 & 0.74 & 2.54 & 2.50 & 2.52 \\
\hline $\mathrm{T}_{6}:$ Jasmonic acid @ $150 \mu \mathrm{M}$ at 50 DAA & 0.69 & 0.71 & 0.70 & 2.08 & 2.34 & 2.21 \\
\hline$T_{7}$ : Salicylic acid @ 50 ppm at 50 DAA and 75 DAA & 0.86 & 0.83 & 0.84 & 2.73 & 2.64 & 2.69 \\
\hline T8 : Salicylic acid@100 ppm at 50 DAA and 75 DAA & 0.86 & 0.84 & 0.85 & 2.72 & 2.67 & 2.69 \\
\hline T9 : Salicylic acid @ 150 ppm at 50 DAA and 75 DAA & 0.89 & 0.84 & 0.87 & 3.15 & 2.76 & 2.95 \\
\hline $\mathrm{T}_{10}$ : Jasmonic acid @ $50 \mu \mathrm{M}$ at 50 DAA and 75 DAA & 0.79 & 0.79 & 0.79 & 2.66 & 2.52 & 2.59 \\
\hline $\mathrm{T}_{11}:$ Jasmonic acid @ $100 \mu \mathrm{M}$ at $50 \mathrm{DAA}$ and 75 DAA & 0.77 & 0.73 & 0.75 & 1.92 & 2.33 & 2.13 \\
\hline $\mathrm{T}_{12}:$ Jasmonic acid @ $150 \mu \mathrm{M}$ at $50 \mathrm{DAA}$ and 75 DAA & 0.76 & 0.72 & 0.74 & 2.11 & 2.36 & 2.23 \\
\hline T13: Control & 0.64 & 0.67 & 0.66 & 1.83 & 2.21 & 2.02 \\
\hline S.Em. \pm & 0.02 & 0.02 & 0.01 & 0.19 & 0.05 & 0.09 \\
\hline C.D. at $5 \%$ & 0.06 & 0.06 & 0.04 & 0.54 & 0.15 & 0.27 \\
\hline
\end{tabular}

DAA: Days after anthesis 
Table.4 Effect of pre-harvest spray of salicylic acid and jasmonic acid on shelf life (Days) in papaya

\begin{tabular}{|c|c|c|c|}
\hline Treatments & $\begin{array}{c}\text { I year } \\
2015-16\end{array}$ & $\begin{array}{c}\text { II year } \\
\text { 2016-17 }\end{array}$ & $\begin{array}{c}\text { Pooled } \\
\text { data }\end{array}$ \\
\hline$T_{1}$ : Salicylic acid @ 50 ppm at 50 DAA & 8.33 & 8.00 & 8.17 \\
\hline$T_{2}$ : Salicylic acid @ 100 ppm at 50 DAA & 8.00 & 8.00 & 8.00 \\
\hline$T_{3}$ : Salicylic acid @ 150 ppm at 50 DAA & 8.67 & 8.67 & 8.67 \\
\hline $\mathrm{T}_{4}:$ Jasmonic acid @ $50 \mu \mathrm{M}$ at 50 DAA & 7.00 & 7.33 & 7.17 \\
\hline$T_{5}:$ Jasmonic acid @ $100 \mu \mathrm{M}$ at 50 DAA & 7.33 & 7.67 & 7.50 \\
\hline $\mathrm{T}_{6}:$ Jasmonic acid @ $150 \mu \mathrm{M}$ at $50 \mathrm{DAA}$ & 7.67 & 7.67 & 7.67 \\
\hline $\mathrm{T}_{7}$ : Salicylic acid @ 50 ppm at 50 DAA and 75 DAA & 9.00 & 9.33 & 9.17 \\
\hline$T_{8}$ : Salicylic acid @ 100 ppm at 50 DAA and 75 DAA & 10.00 & 10.33 & 10.17 \\
\hline $\mathrm{T}_{9}$ : Salicylic acid @ 150 ppm at 50 DAA and 75 DAA & 10.33 & 10.67 & 10.50 \\
\hline $\mathrm{T}_{10}$ : Jasmonic acid @ $50 \mu \mathrm{M}$ at $50 \mathrm{DAA}$ and 75 DAA & 8.33 & 8.33 & 8.33 \\
\hline $\mathrm{T}_{11}:$ Jasmonic acid @ $100 \mu \mathrm{M}$ at $50 \mathrm{DAA}$ and $75 \mathrm{DAA}$ & 8.67 & 9.00 & 8.83 \\
\hline $\mathrm{T}_{12}:$ Jasmonic acid @ $150 \mu \mathrm{M}$ at $50 \mathrm{DAA}$ and $75 \mathrm{DAA}$ & 9.33 & 9.33 & 9.33 \\
\hline $\mathbf{T}_{13}$ : Control & 6.00 & 6.33 & 6.17 \\
\hline S.Em. \pm & 0.27 & 0.35 & 0.24 \\
\hline C.D. at $5 \%$ & 0.79 & 1.01 & 0.70 \\
\hline
\end{tabular}


Table.5 Effect of pre-harvest spray of salicylic acid and jasmonic acid on total soluble soilds ( ${ }^{0}$ Brix) in papaya

\begin{tabular}{|c|c|c|c|c|c|c|c|c|c|c|c|c|}
\hline \multirow[t]{3}{*}{ Treatments } & \multicolumn{12}{|c|}{ Days after harvest } \\
\hline & \multicolumn{3}{|c|}{$3^{\text {rd }}$} & \multicolumn{3}{|c|}{$6^{\text {th }}$} & \multicolumn{3}{|c|}{$9^{\text {th }}$} & \multicolumn{3}{|c|}{$12^{\text {th }}$} \\
\hline & $\begin{array}{c}\text { I year } \\
2015-16\end{array}$ & $\begin{array}{c}\text { II year } \\
2016-17\end{array}$ & $\begin{array}{c}\text { Pooled } \\
\text { data }\end{array}$ & $\begin{array}{c}\text { I year } \\
\text { 2015-16 }\end{array}$ & $\begin{array}{c}\text { II year } \\
2016-17\end{array}$ & $\begin{array}{l}\text { Pooled } \\
\text { data }\end{array}$ & $\begin{array}{c}\text { I year } \\
2015-16\end{array}$ & $\begin{array}{c}\text { II year } \\
\text { 2016-17 }\end{array}$ & $\begin{array}{c}\text { Pooled } \\
\text { data }\end{array}$ & $\begin{array}{c}\text { I year } \\
2015-16\end{array}$ & $\begin{array}{c}\text { II year } \\
2016-17\end{array}$ & $\begin{array}{c}\text { Pooled } \\
\text { data }\end{array}$ \\
\hline $\begin{array}{l}T_{1}: \text { Salicylic acid @ } 50 \\
\text { ppm at } 50 \text { DAA }\end{array}$ & 8.80 & 8.40 & 8.60 & 9.60 & 9.40 & 9.50 & 11.70 & 11.50 & 11.60 & 9.80 & 9.60 & 9.70 \\
\hline $\begin{array}{l}T_{2} \text { : Salicylic acid @ } 100 \\
\text { ppm at } 50 \text { DAA }\end{array}$ & 8.50 & 8.20 & 8.35 & 9.60 & 9.30 & 9.45 & 11.90 & 11.60 & 11.75 & 10.40 & 10.10 & 10.25 \\
\hline $\begin{array}{l}T_{3} \text { : Salicylic acid @ } 150 \\
\text { ppm at } 50 \text { DAA }\end{array}$ & 8.30 & 7.90 & 8.10 & 9.80 & 9.40 & 9.60 & 11.40 & 11.00 & 11.20 & 9.90 & 9.70 & 9.80 \\
\hline $\begin{array}{l}\mathrm{T}_{4} \quad \text { : Jasmonic acid @ } 50 \mu \\
\text { M at } 50 \text { DAA }\end{array}$ & 8.30 & 8.10 & 8.20 & 9.67 & 9.93 & 9.80 & 11.93 & 11.77 & 11.85 & 10.17 & 10.33 & 10.25 \\
\hline $\begin{array}{l}T_{5}: \text { Jasmonic acid @ } 100 \\
\mu \text { M at 50 DAA }\end{array}$ & 8.20 & 8.10 & 8.15 & 9.50 & 9.30 & 9.40 & 11.40 & 11.00 & 11.20 & 10.00 & 9.60 & 9.80 \\
\hline $\begin{array}{l}\mathrm{T}_{6}: \text { Jasmonic acid @ } 150 \\
\mu \text { M at } 50 \text { DAA }\end{array}$ & 8.10 & 7.90 & 8.00 & 9.80 & 9.50 & 9.65 & 11.90 & 11.70 & 11.80 & 10.30 & 10.10 & 10.20 \\
\hline $\begin{array}{l}\mathrm{T}_{7}: \text { Salicylic acid @ } 50 \\
\text { ppm at } 50 \text { DAA and } 75 \text { DAA }\end{array}$ & 8.80 & 8.50 & 8.65 & 10.80 & 10.40 & 10.60 & 12.40 & 12.10 & 12.25 & 10.70 & 10.30 & 10.50 \\
\hline $\begin{array}{l}\mathrm{T}_{8} \quad \text { : Salicylic acid @ } 100 \\
\text { ppm at } 50 \text { DAA and } 75 \text { DAA }\end{array}$ & 9.00 & 8.80 & 8.90 & 10.70 & 10.40 & 10.55 & 12.50 & 12.10 & 12.30 & 10.80 & 10.63 & 10.72 \\
\hline $\begin{array}{l}T_{9} \quad: \text { Salicylic acid @ } 150 \\
\text { ppm at } 50 \text { DAA and } 75 \text { DAA }\end{array}$ & 9.00 & 8.70 & 8.85 & 11.00 & 10.60 & 10.80 & 12.70 & 12.50 & 12.60 & 11.00 & 11.00 & 11.00 \\
\hline $\begin{array}{l}T_{10}: \text { Jasmonic acid @ } 50 \mu \\
M \text { at } 50 \text { DAA and } 75 \text { DAA }\end{array}$ & 8.50 & 8.10 & 8.30 & 10.50 & 10.30 & 10.40 & 12.00 & 11.80 & 11.90 & 10.30 & 10.10 & 10.20 \\
\hline $\begin{array}{l}T_{11}: \text { Jasmonic acid @ } 100 \\
\mu \text { M at } 50 \text { DAA and } 75 \text { DAA }\end{array}$ & 8.40 & 8.20 & 8.30 & 10.10 & 10.00 & 10.05 & 11.80 & 11.50 & 11.65 & 10.20 & 10.10 & 10.15 \\
\hline $\begin{array}{l}T_{12}: \text { Jasmonic acid @ } 150 \\
\mu \text { M at } 50 \text { DAA and } 75 \text { DAA }\end{array}$ & 8.30 & 8.10 & 8.20 & 10.30 & 10.10 & 10.20 & 12.00 & 11.60 & 11.80 & 10.30 & 10.10 & 10.20 \\
\hline $\mathbf{T}_{13}$ : Control & 10.30 & 10.00 & 10.15 & 11.60 & 11.20 & 11.40 & 10.80 & 10.47 & 10.63 & 9.27 & 9.30 & 9.28 \\
\hline S.Em. \pm & 0.15 & 0.17 & 0.13 & 0.1792 & 0.20 & 0.14 & 0.20 & 0.17 & 0.14 & 0.15 & 0.17 & 0.11 \\
\hline C.D. at $5 \%$ & 0.43 & 0.48 & 0.37 & 0.5231 & 0.59 & 0.41 & 0.57 & 0.50 & 0.42 & 0.44 & 0.51 & 0.32 \\
\hline
\end{tabular}

DAA: Days after anthesis 
Salicylic acid also increases potassium content (Farouk et al., 2008) in plant which is useful for maintenance of water flow through stomata, and SA might have increased the number of chloroplasts per cell, number of cells per leaf and consequently more leaf area (Possingham, 1980) which leads to enhanced the efficacy of photosynthetic apparatus with a more potential for resistance against the diseases (Amaresh and Bhatt, 1998). These accumulation of photosynthetic assimilates might have enabled the shoot to meet the nutrient requirement of fruits throughout their development.

A similar increase in the fruit physical parameters by application of salicylic acid was also noticed by Faissal et al., (2014) in keitte mango, Ahmed et al., (2015) in sukkary mango and Gioushy (2016) in Washington navel orange.

\section{Shelf life (days)}

The shelf life recorded in both years and in its pooled mean revealed that highest shelf life of papaya fruits $(10.33,10.67$ and 10.50) was recorded in treatment $\mathrm{T}_{9}$ (SA @ 150 ppm at 50 and 75 DAA) which was at a par withT 8 (SA @ 100 ppm at 50 and 75 DAA) $(10.00$, 10.33 and 10.17). However, control $\left(\mathrm{T}_{13}\right)$ recorded minimum shelf life $(6.00,6.33$ and 6.17) (Table 4).

The increase in shelf life due to salicylic acid application might be attributed to its role in suppression in respiration and reduction in transpiration rates of papaya fruits by closing the stomata of treated fruits, which otherwise could lead to increased weight loss. Moreover, application of salicylic acid enhanced firmness of fruits (Srivastava and Dwivedi, 2000) by suppressing the activity of cell wall degrading enzyme like pectinmethyl esterase, cellulase, polygalactronase, xylanse, and $\beta-1-3$ glucanase (Ali et al., 2004).
Production of ethylene was delayed by interference of Salicylic acid (Raskin, 1992) thereby increased the shelf life. Such delay in ethylene production might be associated with the decreased 1-Aminocyclopropane-1Carboxylic Acid (ACC) synthase and or ACC oxidase activity as reported in Banana (Srivastava and Dwivedi, 2000).

\section{Total soluble solids $\left({ }^{\circ} \mathrm{Brix}\right)$}

A gradual increase in T.S.S $\left({ }^{\circ}\right.$ Brix) during the storage period was observed in all the treatments upto $9^{\text {th }}$ day of storage it was decreased later on. However, in control $\left(\mathrm{T}_{13}\right)$ higher TSS was recorded at 6 days after storage and showed a declining trend later on. This could be due to the faster ripening process in control which was regulated by the application of salicylic acid and jasmonic acid. On $12^{\text {th }}$ day of storage highest TSS was $\left(11.00{ }^{\circ}\right.$ Brix) was observed in the treatment $\mathrm{T}_{9}$ which was at a par with $\mathrm{T}_{8}\left(10.72{ }^{\circ} \mathrm{Brix}\right)$. At $9^{\text {th }}$ day after storage even the jasmonic acid applied at different concentrations improved the total soluble solids of the fruits compared to control (Table 5).

The increase of TSS by salicylic acid might be due to regulation of plant growth by enhancing the "Rubisco" activity and also enhanced the sugar translocation from leaves to the developing fruit by improving the $\alpha$ amylase activity by salicylic acid (Sharma $e t$ al., 1986). The hydrolysis of sucrose by invertase regulates by the levels of some plant hormones like IAA, salicylic acid and jasmonic acid (Leclere et al., 2003).

\section{References}

Ahmed, F.F., Mansour, A.E.M and Merwad, M.A. 2015. Physiological studies on the effect of spraying Salicylic acid on fruiting of succary mango trees. International Journal of ChemTech 
Research. 8(4): 2142-2149.

Ali, Z., Chin, L.H and Lazan, H. 2004. A comparative study on wall degrading enzymes, pectin modifications and softening during ripening of selected tropical fruits. Plant Sciences. 167: 317-327.

Amaresh, C and Bhatt, R.K. 1998. Biochemical and physiological response to salicylic acid in reaction to systemic acquired resistance. Photosynthetica. 35(2): 255-258.

Buta, I.T and Moilne, H.E. 1998. Methyl jasmonate extends shelf life and reduces microbial contamination of fresh-cut celery and peppers. Journal of Agricultural and Food Chemistry. 46: 1253-1256.

Faissal, F., Ahmed Mohamed, Kamel K.h. and Hamdy, M. 2014. The Synergistic Effects of Using Plant Extracts and Salicylic Acid on Yield and Fruit Quality of Keitte Mango Trees. Stem Cell. 5(2): 30-39.

Farouk S., Ghoneem K.M and Abeer A. Ali. 2008. Induction and Expression of systematic resistance to downy mildew disease in cucumber plant by elicitors. Egyptian Journal of Phytopathology. (1-2): 95-111.

Farouk, S and Osman, M.A. 2011. The effect of plant defense elicitors on common bean (Phaseolus vulgaris L.) growth and yield in absence or presence of spider mite (Tetranychus urticae Koch) infestation. Journal of Stress Physiology and Biochemistry. 7(3): 5-22.

Gioushy, S.F.E. 2016. Productivity, fruit quality and nutritional status of Washington navel orange trees as influenced by foliar application with salicylic acid and potassium silicate combinations. Journal of Horticultural Science and Ornamental Plants. 8 (2): 98-107.

India stat, 2016-17., www.Indiastat.com.

Leclere, S., Scmelz, E.A and Chourey, P.S. 2003. Cell wall invertase-deficient miniature kernels have altered phytohormones levels. Phytochemistry. 69(3): 692-699.

Possingham, J.V. 1980 Plastid replication and development in the life cycle of higher plants. Annual Review of Plant Physiology. 31:113-129.

Raskin, I. 1992. Salicylate, a new plant hormone. Plant Physiology. 99:799803.

Sharma, S., Sharma, S.S and Rau, V.K. 1986. Reversal by phoenolic compounds of abscussic acid-induced inhibition of in vitro activity of amylase from seeds of Triticum aestivum L. New Phytologist. 103(2): 293-297.

Srivastava M.K., and Dwivedi, U.N. 2000. Delayed ripening of banana fruit by salicylic acid. Plant Science. 158: 8796.

\section{How to cite this article:}

Sreedhar Devarakonda, C. Madhumathi, V. Umamahesh, L. Mukunda Lakshmi, M. Lakshmi Narayana Reddy, V. Vijaya Bhaskar and Rajasekharam, T. 2020. Physiological Changes and Shelf-Life Extension of Papaya (Carica papaya L.) cv. Red Lady as Influenced by Pre-Harvest Spray of Plant Elicitors. Int.J.Curr.Microbiol.App.Sci. 9(05): 2599-2608. doi: https://doi.org/10.20546/ijcmas.2020.905.297 